\title{
An Overview of COVID-19 Testing and Implications for Otolaryngologists
}

\author{
Xiao Zhao, MD, $\mathrm{PhD}^{1}$ \\ ${ }^{1}$ The University of Texas MD Anderson Cancer Center
}

May 7, 2020

\begin{abstract}
Background : Testing for SARS-CoV-2 is important for decision making prior to surgery in otolaryngology. An understanding of current and developing testing methods is important for interpreting test results.

Methods : We performed a literature review of current evidence surrounding SARS-CoV-2 diagnostic testing highlighting its utility, limitations, and implications for otolaryngologists.

Results : The currently accepted RT-PCR test for SARS-CoV-2 has varying sensitivity according to which subsite of the aerodigestive tract is sampled. Nasal swab sensitivities appear to be about $70 \%$. Chest CT imaging for screening purposes is not currently recommended.

Conclusion : Due to the current sensitivity of RT-PCR based testing for SARS-CoV-2, a negative test cannot rule out COVID19. Full PPE should be worn during high risk procedures such as aerosol generating procedures even if testing is negative. Patients who test positive during screening should have their surgeries postponed if possible until asymptomatic and have tested negative for SARS-CoV-2.
\end{abstract}

Daniel B Vinh MD 1 , Xiao Zhao MD PhD*,2, Kimberley L Kiong MBBS 2 , Theresa Guo MD 2 , Yelda Jozaghi MD 2 , Chris Yao MD 2 , James M Kelley MD PhD 3 , Ehab Hanna MD 2

1 Department of Otolaryngology - Head and Neck Surgery, Baylor College of Medicine, Houston, TX, USA 2 Department of Head and Neck Surgery, MD Anderson Cancer Center, Houston, TX, USA 3 Department of Laboratory Medicine, Division of Pathology and Laboratory Medicine, MD Anderson Cancer Center, Houston, TX, USA

* Corresponding author. E-mail: XZhao6@mdanderson.org

Key words: COVID-19, PCR, sensitivity, SARS-CoV-2, testing

\begin{abstract}
Background: Testing for SARS-CoV-2 is important for decision making prior to surgery in otolaryngology. An understanding of current and developing testing methods is important for interpreting test results.

Methods : We performed a literature review of current evidence surrounding SARS-CoV-2 diagnostic testing highlighting its utility, limitations, and implications for otolaryngologists.

Results : The currently accepted RT-PCR test for SARS-CoV-2 has varying sensitivity according to which subsite of the aerodigestive tract is sampled. Nasal swab sensitivities appear to be about $70 \%$. Chest CT imaging for screening purposes is not currently recommended.
\end{abstract}


Conclusion: Due to the current sensitivity of RT-PCR based testing for SARS-CoV-2, a negative test cannot rule out COVID-19. Full PPE should be worn during high risk procedures such as aerosol generating procedures even if testing is negative. Patients who test positive during screening should have their surgeries postponed if possible until asymptomatic and have tested negative for SARS-CoV-2.

\section{Introduction}

In December of 2019, an outbreak of patients with severe pneumonia was reported in Wuhan, China. A novel Coronavirus was isolated as the causative agent. It has been named by the World Health Organization (WHO) as the severe acute respiratory distress syndrome coronavirus 2 (SARS-CoV-2) which causes the coronavirus disease 2019 (COVID-19). The genome of this novel coronavirus is a single-stranded positivesense RNA (+ssRNA). It belongs to the family Coronaviridae containing strains responsible for the SARS outbreak in 2003 as well as the Middle East respiratory syndrome (MERS) outbreak in 2012. SARS-CoV-2 has spread rapidly across the world and by March $11^{\text {th }}$, 2020, the WHO officially declared the outbreak a pandemic. ${ }^{4}$ This pandemic has resulted in unprecedented challenges to the healthcare system and to society as a whole.

Among the healthcare workforce, otolaryngologists are at particular risk for acquiring the disease due to performance of exams and procedures involving a potentially infected upper aerodigestive tract. Routine endoscopic examinations including nasal endoscopy, flexible laryngoscopy as well as the use of energy devices during surgery are considered aerosol generating procedures (AGPs) with high risk for transmission. ${ }^{5}$ An analysis of 138 patients hospitalized with COVID-19 in Wuhan, China found that 40 (29\%) were healthcare workers. ${ }^{6}$ In fact, the first reported physician death associated with the disease was that of an otolaryngologist in Wuhan. ${ }^{7}$ In recognition of these risks and to conserve limited resources, the American Academy of Otolaryngology-Head and Neck Surgery has recommended only performing procedures or surgeries that are time sensitive or emergent. ${ }^{8}$ Similar recommendations have been made by the American College of Surgeons as well as the Centers for Medicare and Medicaid Services (CMS) $\cdot{ }^{9,10}$ In an effort to reduce risk, many professional societies have recommended screening for COVID-19 prior to high risk procedures as emerging data suggests that patients can be asymptomatic carriers. ${ }^{5,11}$

In this article, we will review the current testing standards for COVID-19 and discuss their strengths and limitations. Of note, new information on the COVID-19 pandemic is being published at a fast rate. The data presented here rely primarily on early studies with confounders that can influence interpretation. As there is no current gold standard for SARS-CoV-2 testing, careful re-evaluation of the published evidence over time will be imperative.

\section{Presenting symptoms and lab values}

The first report of 41 patients confirmed to have COVID-19 in Wuhan, China found that initial symptoms included fever $(98 \%)$, cough $(76 \%)$, myalgia or fatigue (44\%), sputum production (28\%), headache (8\%), hemoptysis (5\%), and diarrhea (3\%). Dyspnea developed in 55\% of patients with progression to ARDS in $29 \%$ of patients. A total of $13(32 \%)$ patients were admitted to the ICU and 6 patients (15\%) died. ${ }^{12}$ A subsequent report of 138 patients, also from Wuhan, found that $98.6 \%$ of patients had fever, $69.6 \%$ had fatigue, and $59.4 \%$ dry cough. In that cohort, 36 patients $(26 \%)$ required ICU care, and 6 patients $(4.3 \%)$ died. ${ }^{6}$ A larger, multi-institutional characterization of 1099 patients with laboratory confirmed COVID-19 throughout China found that $88.7 \%$ of patients developed fever during their hospital admission. The second most common symptom was cough (67.8\%). Less frequent were nausea or vomiting (5\%) and diarrhea (3.8\%). Upper respiratory type symptoms were relatively infrequent with only $13.9 \%$ presenting with sore throat and $4.8 \%$ of patients with nasal congestion. Of those patients, $5 \%$ were admitted to the ICU, $2.3 \%$ required mechanical ventilation, and $1.4 \%$ died. ${ }^{13}$ While not initially reported, multiple recent studies have found chemosensory dysfunctions associated with COVID-19, ${ }^{14,15}$ with one study finding up to $85.6 \%$ and $88 \%$ showing olfactory and gustatory dysfunction respectively. ${ }^{16}$ Notably, emerging data has found the asymptomatic carrier rate to be in the range of $17.9-21.7 \% .{ }^{17,18}$ The most common laboratory abnormalities included lymphocytopenia $(83.2 \%)$, thrombocytopenia $(36.2 \%)$, and leukopenia $(33.7 \%) .{ }^{13}$ 


\section{Current Testing Standards}

The current test for the diagnosis of COVID-19, as recommended by the United States Centers for Disease Control and Prevention (CDC), is a qualitative real time RT-PCR nasopharyngeal swab which detects the presence of specific segments of the SARS-CoV-2 genome. The current CDC test detects and amplifies two regions of the nucleoside coding genome, N1 and N2. A positive test is found when both segments are detected. An inconclusive test occurs when only 1 segment is detected, and a negative test occurs when both segments are not detected. RNA primers for amplification and detection of these genetic segments are available publicly on the CDC website. ${ }^{19}$ Internal testing by the CDC comparing these primers and probes for N1 and N2 against public domain nucleotide sequences found no significant homologies with the human genome, other coronaviruses, or human microflora that would lead to a potential false positive. ${ }^{20}$ Limits of detection by RT-PCR were also tested internally by the CDC. While detection thresholds varied according to different commercially available master mixes of enzyme, all assays were able to reliably detect concentrations of 10 copies/uL. ${ }^{20}$ Multiple laboratories within the United States including reference laboratories and private hospital groups have implemented this testing, made allowable by the FDA under an Emergency Use Authorization (EUA). The FDA has also approved an EUA for multiple other commercial entities and academic medical centers to offer molecular testing either modified from the CDC protocol or developed with unique primers. ${ }^{21}$ RT-PCR testing by other countries has targeted other sequences of the SARS-CoV-2 genome. ${ }^{22}$

\section{Sensitivity of PCR Assay}

Currently the CDC states that a negative result does not exclude SARS-CoV-2 infection. Sources of false negative testing include patient misidentification, collection of inappropriate or inadequate material, improper specimen transportation, low viral density in pre-symptomatic patients, and lab errors. In addition to these factors, the location of testing in the aerodigestive tract plays a large role in the sensitivity to detect SARS-CoV-2. As was shown by Zou et al., ${ }^{3}$ viral loads in the upper respiratory tract of 18 patients varied according to sub-site, with about 64 fold higher viral loads detected in the nasal cavity than in the pharynx. In a study of 213 patients with confirmed COVID-19, the authors found that sputum samples showed the highest positive rate in both severe (88.9\%) and mild (82.2\%) cases, followed by nasal swabs $(73.3 \%, 72.1 \%)$, and then throat swabs $(60.0 \%, 61.3 \%) .^{2}$ In a similar study consisting of 205 patients, Wang et al. found that bronchoalveolar lavage had the highest positive rates $(93 \%)$, followed by sputum (72\%), nasal swabs (63\%), bronchoscope brushings (46\%), pharyngeal swabs (32\%), feces (29\%), and blood (1\%). ${ }^{1}$ From these studies, it appears that the highest positive detection rate is from lower respiratory tract specimens. A plausible explanation is that SARS-CoV-2 binds to human angiotensin-converting enzyme 2 (ACE2), found predominantly in the lower respiratory tract. ${ }^{23}$ However a potential confounder is that invasive lower respiratory tract sampling would be primarily performed on patients that have been intubated, which suggests that these patients overall may have higher viral load. Finally, to add further complexity to diagnosis, there may be variable viral load and shedding over time, even when the patient is asymptomatic. There currently is no consensus on when PCR testing should be performed, and this is an area that needs further study.

\section{Imaging}

Chest CT imaging of COVID-19 patients typically demonstrate ground-glass opacities, multifocal patchy consolidation, and/or interstitial changes with a peripheral distribution. ${ }^{24}$ In one study, the authors found that Chest CT scans had higher sensitivity for diagnosis of COVID-19 (88\%) as opposed to initial RTPCR using throat swabs (59\%). ${ }^{25}$ Another study assessed radiologists' ability to distinguish COVID-19 pneumonia from non-COVID-19 pneumonia. It found sensitivities among radiologists to be between 73-93\% and specificities to be between $93-100 \% .{ }^{26}$ At this time, however, the majority of radiology societies do not recommend routine screening for COVID-19 with chest $\mathrm{CT} .{ }^{27}$

\section{Serologic Testing}

Testing of IgM and IgG specific antibodies to SARS-CoV-2 is done readily by obtaining blood samples and does not require specialized PCR equipment. An initial study on the kinetics of antibody formation 
found that of 535 patients, the total seroconversion rate for total Ab, IgM, and IgG was $93.1 \%, 82.7 \%$, and $64.7 \%$, with median seroconversion time of 11,12 , and 14 days. ${ }^{28}$ The same authors reported that combining RT-PCR and antibody detection can improve the sensitivity for diagnosis of COVID-19. ${ }^{28}$

A patient with a positive serologic test, however, only means that they have been exposed to SARS-CoV-2. Whether the resulting antibodies confer immunity to the virus requires further study. As such, the presence of antibodies does not necessarily reflect a person's potential to transmit the disease. There are reports showing that patients who developed COVID-19 and then resolved with negative serial PCR testing later again became positive on PCR assay. ${ }^{29}$ This could potentially represent a false positive result, or a viral carrier state despite a measured serologic conversion. As such, while serologic testing is important in the setting of confirming SARS-CoV-2 infection, this test does not indicate whether the patient is or is not actively infectious and shedding the virus. Until further research is done, the WHO does not currently recommend utilizing serologic testing to guide decision making. ${ }^{30}$

\section{How Testing Impacts Management}

For patients who are being screened prior to potential head and neck surgery, a nasopharyngeal swab should be performed by a healthcare provider, preferably 24 hours prior to surgery. ${ }^{31}$ In an effort to broaden access to testing, the CDC has now allowed self-collection of nasal swabs. ${ }^{32}$ While there are no data on the sensitivity of these self-administrated tests, there is potentially lower sensitivity due to inadequate sampling. Until more data is available, self-administered testing has not been established as an adequate alternative to testing performed by a health care provider.

Given the above sensitivity limitations of PCR-based assays, it is not clear whether a negative COVID-19 test should impact practice patterns in a high-risk specialty such as Otolaryngology-Head and Neck Surgery. Current sensitivities are considered to be acceptable for the general population and for patients who do not undergo high-risk procedures. However, for patients who undergo AGPs, the impact of a false negative result has serious implications to healthcare providers and other patients in the hospital. As such, many institutions have recommended that all personnel at risk for exposure to aerosolization of SARS-CoV-2 wear full personal protective equipment. This would include N95 or powered air purifying respirator (PAPR), even for asymptomatic patients with negative testing during this pandemic. ${ }^{7}$

For patients who test positive for COVID-19, the CDC has recommended that these patients be quarantined for at least 7 days and be asymptomatic with two negative PCR results done 24 hours apart to confirm clearance of SARS-CoV-2. ${ }^{33}$ New evidence has emerged suggesting that performing elective surgery on patients with COVID-19 carries high risk of patient morbidity. Of 34 patients who underwent elective surgeries and subsequently became positive for COVID-19, $44 \%$ required admission to the ICU and the mortality rate was 20.5\% ${ }^{34}$ As such, all patients with confirmed COVID-19 should be discussed within a multi-disciplinary team and surgery deferred, if possible, until proof of virus clearance is obtained and the patient is asymptomatic.

\section{Conclusion}

Currently, there is no gold standard for the diagnosis of SARS-CoV-2. Early evidence suggests that the current standard for the diagnosis of SARS-CoV-2 using RT-PCR has variations in sensitivity dependent on the sub-site tested. Nasal swab specimens appear to have high viral loads, but even then sensitivities are in the range of $60-70 \% \cdot{ }^{1-3}$ Furthermore, while clinical history, CT imaging, and laboratory tests may support the diagnosis of COVID-19, none of these tests alone or in combination with RT-PCR have been proven to have optimal sensitivity to rule out SARS-CoV-2 infection, especially for high risk AGPs. As such even for patients tested negative for COVID19, many institutions suggest that the highest level of PPE available should be worn that protects against potential transmission of aerosolized SARS-CoV-2 for procedures of the upper aerodigestive tract. Moreover, the emerging data demonstrating high morbidity and mortality for patients who develop COVID-19 after elective surgeries would suggest that elective surgery should be postponed in this patient population. Finally, it is imperative to point out once again the evidence on the COVID-19 pandemic is rapidly evolving. We will need to constantly re-evaluate the nature of testing and result interpretation, especially as the prevalence of SARS-CoV-2 continues to change and new testing 
methodologies become available.

References

1. Wang W, Xu Y, Gao Ret al. Detection of SARS-CoV-2 in Different Types of Clinical Specimens. JAMA 2020 .

2. Yang Y, Yang M, Shen Cet al. Evaluating the accuracy of different respiratory specimens in the laboratory diagnosis and monitoring the viral shedding of 2019-nCoV infections. medRxiv 2020:2020.2002.2011.20021493.

3. Zou L, Ruan F, Huang Met al. SARS-CoV-2 Viral Load in Upper Respiratory Specimens of Infected Patients. New England Journal of Medicine 2020; 382:1177-1179.

4. WHO Timeline - COVID-19. Available at: https://www.who.int/news-room/detail/08-04-2020-whotimeline - covid-19. 2020.

5. Givi B, Schiff BA, Chinn SBet al. Safety Recommendations for Evaluation and Surgery of the Head and Neck During the COVID-19 Pandemic. JAMA Otolaryngology-Head \& Neck Surgery 2020.

6. Wang $\mathrm{D}, \mathrm{Hu} \mathrm{B}, \mathrm{Hu}$ Cet al. Clinical Characteristics of 138 Hospitalized Patients With 2019 Novel Coronavirus-Infected Pneumonia in Wuhan, China. JAMA 2020; 323:1061.

7. Chan JYK, Wong EWY, Lam W. Practical Aspects of Otolaryngologic Clinical Services During the 2019 Novel Coronavirus Epidemic. JAMA Otolaryngology-Head \& Neck Surgery 2020.

8. New Recommendations Regarding Urgent and Nonurgent Patient Care. Available at: https://www.entnet.org/content/new-recommendations-regarding-urgent-and-nonurgent-patient-care.

9. COVID-19: Recommendations for Management of Elective Surgical Procedures. Available at: https://www.facs.org/covid-19/clinical-guidance/elective-surgery.

10. Non-emergent, Elective Medical Services, and Treatment Recommendations. Available at: https://www.cms.gov/files/document/cms-non-emergent-elective-medical-recommendations.pdf.

11. Bai Y, Yao L, Wei Tet al. Presumed Asymptomatic Carrier Transmission of COVID-19. JAMA 2020.

12. Huang C, Wang Y, Li Xet al. Clinical features of patients infected with 2019 novel coronavirus in Wuhan, China. The Lancet 2020; 395:497-506.

13. Guan W-J, Ni Z-Y, Hu Yet al. Clinical Characteristics of Coronavirus Disease 2019 in China. New England Journal of Medicine 2020.

14. Vaira LA, Salzano G, Deiana G, De Riu G. Anosmia and ageusia: common findings in COVID-19 patients. The Laryngoscope 2020.

15. Yan CH, Faraji F, Prajapati DP, Boone CE, Deconde AS. Association of chemosensory dysfunction and Covid-19 in patients presenting with influenza-like symptoms. International Forum of Allergy \& Rhinology 2020 .

16. Lechien JR, Chiesa-Estomba CM, De Siati DRet al. Olfactory and gustatory dysfunctions as a clinical presentation of mild-to-moderate forms of the coronavirus disease (COVID-19): a multicenter European study. European Archives of Oto-Rhino-Laryngology 2020.

17. Song H, Xiao J, Qiu Jet al. A considerable proportion of individuals with asymptomatic SARS-CoV-2 infection in Tibetan population. medRxiv 2020:2020.2003.2027.20043836.

18. Mizumoto K, Kagaya K, Zarebski A, Chowell G. Estimating the asymptomatic proportion of coronavirus disease 2019 (COVID-19) cases on board the Diamond Princess cruise ship, Yokohama, Japan, 2020. Eurosurveillance 2020; 25. 
19. Research Use Only 2019-Novel Coronavirus (2019-nCoV) Real-time RT-PCR Primer and Probe Information. Available at: https://www.cdc.gov/coronavirus/2019-ncov/lab/rt-pcr-panel-primer-probes.html.

20. CDC 2019-Novel Coronavirus (2019-nCoV) Real-Time RT-PCR Diagnostic Panel. Available at: https://www.fda.gov/media/134922/download.

21. Emergency Use Authorization. Available at: https://www.fda.gov/emergency-preparedness-andresponse/mcm-legal-regulatory-and-policy-framework/emergency-use-authorization

22. Jung YJ, Park G-S, Moon JHet al. Comparative analysis of primer-probe sets for the laboratory confirmation of SARS-CoV-2: Cold Spring Harbor Laboratory, 2020.

23. Paules CI, Marston HD, Fauci AS. Coronavirus Infections-More Than Just the Common Cold. JAMA 2020; 323:707.

24. Chung M, Bernheim A, Mei Xet al. CT Imaging Features of 2019 Novel Coronavirus (2019-nCoV). Radiology 2020; 295:202-207.

25. Ai T, Yang Z, Hou Het al. Correlation of Chest CT and RT-PCR Testing in Coronavirus Disease 2019 (COVID-19) in China: A Report of 1014 Cases. Radiology 2020:200642.

26. Bai HX, Hsieh B, Xiong Zet al. Performance of radiologists in differentiating COVID-19 from viral pneumonia on chest CT. Radiology 2020:200823.

27. Simpson S, Kay FU, Abbara Set al. Radiological Society of North America Expert Consensus Statement on Reporting Chest CT Findings Related to COVID-19. Endorsed by the Society of Thoracic Radiology, the American College of Radiology, and RSNA. Radiology: Cardiothoracic Imaging 2020; 2:e200152.

28. Zhao J, Yuan Q, Wang Het al. Antibody responses to SARS-CoV-2 in patients of novel coronavirus disease 2019. Clinical Infectious Diseases 2020.

29. Lan L, Xu D, Ye Get al. Positive RT-PCR Test Results in Patients Recovered From COVID-19. JAMA 2020 .

30. Advice on the use of point-of-care immunodiagnostic tests for COVID-19. Available at: https://www.who.int/news-room/commentaries/detail/advice-on-the-use-of-point-of-careimmunodiagnostic-tests-for-covid-19\#.XpM1L2aCbKI.email.

31. Rusha J. Patel AK, Caitlin McMullen. Early Institutional Head and Neck Oncologic and Microvascular Surgery Practice Patterns Across the United States During the SARS-CoV-2 (COVID19) Pandemic. Authorea 2020.

32. Interim Guidelines for Collecting, Handling, and Testing Clinical Specimens from Persons for Coronavirus Disease 2019 (COVID-19). Available at: https://www.cdc.gov/coronavirus/2019-nCoV/lab/guidelinesclinical-specimens.html.

33. Discontinuation of Isolation for Persons with COVID-19 Not in Healthcare Settings (Interim Guidance). Available at: https://www.cdc.gov/coronavirus/2019-ncov/hcp/disposition-in-home-patients.html.

34. Lei S, Jiang F, Su Wet al. Clinical characteristics and outcomes of patients undergoing surgeries during the incubation period of COVID-19 infection. EClinicalMedicine 2020:100331. 